\title{
Assessment of body fluid in critically ill patients with acute kidney injury requiring continuous renal replacement therapy
}

\author{
Jae Seok Kim ${ }^{(i)}$, Byoung-Geun Han ${ }^{(i)}$ \\ Department of Nephrology, Yonsei University Wonju College of Medicine, Wonju, Republic of Korea
}

Previous studies have presented prognostic factors for patients with acute kidney injury (AKI) who require continuous renal replacement therapy (CRRT), including elderly age, systemic inflammation, oliguria, fluid imbalance, comorbidities, and need for mechanical ventilation [1-4]. Nevertheless, prognostic factors that accurately predict outcomes have not been determined. Among the prognostic factors under consideration, there is growing interest in the clinical role of negative or positive fluid balance associated with fluid resuscitation [5]. Most importantly, an ideal method of body fluid assessment should be an easy, objective, and reproducible evaluation, and a treatment plan based on the results should confirm whether it affects the outcomes of the patient.

The pathogenesis of AKI in critical illness is multi-factorial and complex. Therefore, therapeutic interventions for patients with AKI remain heterogeneous. In particular, it can be said that it is representative issues to determine an appropriate fluid administration dose and fluid removal amount, and the timing of renal replacement therapy.

Received November 18, 2020; Accepted November 19, 2020

Editor: Tae-Hyun Yoo, Yonsei University, Seoul, Republic of Korea Correspondence: Byoung-Geun Han

Department of Nephrology, Yonsei University Wonju College of Medicine, 20 Ilsan-ro, Wonju 26426, Republic of Korea. E-mail: neptune@yonsei.ac.kr

Copyright (C) 2020 by The Korean Society of Nephrology

(a) This is an open-access article distributed under the terms of the Creative Commons Attribution Non-Commercial License (http://creativecommons. org/licenses/by-nc-nd/4.0/), which permits unrestricted non-commercial use, distribution, and reproduction in any medium, provided the original work is properly cited.
Fluid overload is strongly associated with adverse clinical outcomes and may contribute to development and persistence of AKI in critically ill patients [5]. Assessment of volume status in patients with AKI requiring renal replacement therapy is important not only for maintaining hemodynamic stability, but also for improving the outcomes of AKI. However, the traditional methods for detecting degree and severity of fluid imbalance have several limitations. Furthermore, there is no clinical gold standard that can be used as a guide for fluid administration to prevent fluid overload in patients with AKI.

Many studies have consistently highlighted the clinical significance of fluid overload in critically ill patients. Jhee et al [6] also showed a relationship between fluid overload and mortality in elderly patients with AKI requiring CRRT. Their multicenter prospective cohort study clearly showed that critically ill patients with positive fluid balance for 72 hours prior to CRRT initiation were associated with higher mortality compared to those without. However, despite such clear results, attention should be paid to important issues in their research.

First, as the researchers noted, it is important to define fluid overload using a decisive rather than arbitrary method. Various methods such as clinical examination, serial weights, cumulative fluid balance (CFB), chest Xray, lung ultrasound, echocardiography, and bioimpedance analysis can be used to evaluate volume status, but they all have limitations. Therefore, because there is no rapid, objective, non-invasive, and relatively affordable method to quantitatively assess volume status, it is difficult to set a treatment policy for critically ill patients with AKI requiring CRRT. Unlike the study by Jhee et al [6], some have defined the criteria for fluid overload as a 
greater than $10 \%$ increase in body weight relative to baseline $[7,8]$. Furthermore, the relationship between fluid overload based on this criterion and clinical outcome has been well elucidated.

Second, the efforts to avoid positive fluid balance in initial treatment of critically ill patients as proposed by Jhee et al [6] may conflict with current intensive care strategies. Since early goal-directed therapy for patients who present to the emergency department with severe sepsis, septic shock, or sepsis syndrome was proposed by Rivers et al [9], clinical therapeutic strategies based on early fluid resuscitation to restore or maintain intravascular volume have been useful [10]. The recently updated version of the Surviving Sepsis Campaign proposes a rapid infusion of crystalloid solution at a minimum dose of $30 \mathrm{~mL} /$ $\mathrm{kg}$ in the initial critical phase [11]. Although this therapeutic strategy has been recommended mainly in cases of hypotension or lactic acidosis, early fluid resuscitation is currently performed for most critically ill patients.

Third, given that not all patients underwent standardized early fluid resuscitation from treatment initiation, setting the reference point for fluid overload using cumulative fluid data assessed at 72 hours before CRRT initiation is limited, even though the aforementioned CFB is widely accepted as a method to document fluid balance. We cannot rule out the possibility that positive fluid balance might have been the result of early fluid resuscitation due to hemodynamic instability of the critically ill patients in the study. In addition, even though propensity score matching and adjustment for confounding factors were performed, it is unclear whether hemodynamic stability was properly controlled between the two groups because the authors did not provide data on use of inotropic agents or vasoactive agents. Therefore, the association between fluid overload and mortality may largely be secondary.

Fourth, an additional measurement method for determining the appropriate amount of fluid should have been used. This was mentioned as one of the limitations of the study. The definition of CFB is the sum of each day's fluid balance over a period of time. Therefore, it cannot be assumed that a positive fluid balance is accompanied by fluid overload. Furthermore, CFB is often inaccurate, and insensible fluid losses are not reflected. Bioimpedance analysis may be a good replacement, even though it has not been verified in critically ill patients with AKI [12].
In conclusion, the study by Jhee et al [6] is valuable because it supports the existing research that fluid overload is related to poor clinical outcomes in critically ill patients. Preventing fluid overload should accompany accurate assessment of individual volume status. Future studies should provide more scientific and accurate analysis methods, allowing innovation in evaluation of volume status in critically ill patients with AKI.

\section{Conflicts of interest}

All authors have no conflicts of interest to declare.

\section{Authors' contributions}

Jae Seok Kim provided intellectual content of critical importance to the work. Byoung-Geun Han participated in the conception and wrote the manuscript. All authors read and approved the final manuscript.

\section{References}

[1] Oh HJ, Shin DH, Lee MJ, et al. Urine output is associated with prognosis in patients with acute kidney injury requiring continuous renal replacement therapy. J Crit Care 2013; 28:379-388.

[2] Park JY, An JN, Jhee JH, et al. Early initiation of continuous renal replacement therapy improves survival of elderly patients with acute kidney injury: a multicenter prospective cohort study. Crit Care 2016;20:260.

[3] Kim H, Kim J, Seo C, et al. Body mass index is inversely associated with mortality in patients with acute kidney injury undergoing continuous renal replacement therapy. Kidney Res Clin Pract 2017;36:39-47.

[4] Zarbock A, Kellum JA, Schmidt C, et al. Effect of early vs delayed initiation of renal replacement therapy on mortality in critically ill patients with acute kidney injury: the ELAIN randomized clinical trial. JAMA 2016;315:2190-2199.

[5] Prowle JR, Kirwan CJ, Bellomo R. Fluid management for the prevention and attenuation of acute kidney injury. Nat Rev Nephrol 2014;10:37-47.

[6] Jhee JH, Park JY, An JN, et al. Cumulative fluid balance and mortality in elderly patients with acute kidney injury requiring continuous renal replacement therapy: a multicenter prospective cohort study. Kidney Res Clin Pract 2020;39:414-425. 
[7] Bouchard J, Soroko SB, Chertow GM, et al.; Program to Improve Care in Acute Renal Disease (PICARD) Study Group. Fluid accumulation, survival and recovery of kidney function in critically ill patients with acute kidney injury. Kidney Int 2009;76:422-427.

[8] Samoni S, Vigo V, Reséndiz LI, et al. Impact of hyperhydration on the mortality risk in critically ill patients admitted in intensive care units: comparison between bioelectrical impedance vector analysis and cumulative fluid balance recording. Crit Care 2016;20:95.

[9] Rivers E, Nguyen B, Havstad S, et al.; Early Goal-Directed Therapy Collaborative Group. Early goal-directed therapy in the treatment of severe sepsis and septic shock. $N$ Engl $J$
Med 2001;345:1368-1377.

[10] Malbrain MLNG, Van Regenmortel N, Saugel B, et al. Principles of fluid management and stewardship in septic shock: it is time to consider the four D's and the four phases of fluid therapy. Ann Intensive Care 2018;8:66.

[11] Levy MM, Evans LE, Rhodes A. The surviving sepsis campaign bundle: 2018 update. Intensive Care Med 2018;44: 925-928.

[12] Basso F, Berdin G, Virzì GM, et al. Fluid management in the intensive care unit: bioelectrical impedance vector analysis as a tool to assess hydration status and optimal fluid balance in critically ill patients. Blood Purif 2013;36:192-199. 